Die Geschichte des Zürcher Medizinstudiums steht im Mittelpunkt des Sammelbands «Innenansichten einer Ärzteschmiede», der anlässlich des Universitätsjubiläums im ChronosVerlag erschienen ist. Die Schweizerische Ärztezeitung druckt Auszüge aus den Artikeln in einer Serie ab. Unter dem Titel «Vom Grünschnabel zum Weisskittel» zeigt das Medizinhistorische Archiv zudem bis 31. Mai eine Ausstellung zum Thema im Hauptge- bäude der Universität (www.175jahre.uzh.ch/ gruenschnabel-weisskittel). Der Beitrag, aus dem die heutigen Auszüge stammen, untersucht die erste Generation Schweizer Juden der Universität Zürich, die aus den beiden Surbtaler Gemeinden Endingen und Lengnau (AG) stammten und über das Medizinstudium einen Weg aus der sozialen und geografischen Beengung fanden.

\title{
Die ersten Schweizer Juden als Medizinstudenten in Zürirch*
}

\section{Daniel Teichman}

* Auszüge aus dem gleichnamigen Beitrag des Autors in: Ritzmann I, Schweer W, Wolff E (Hrsg.). Innenansichten einer Ärzteschmiede. Lehren, lernen und leben - aus der Geschichte des Zürcher Medizinstudiums. Zürich, Chronos; 2008. 240 S., 80 teils farbige Abbildungen. Fr. 34.-/€ 20.60. ISBN 978-3-0340-0909-6. Quellenverweise im Originalartikel.

Korrespondenz:

Daniel Teichman

Facharzt für Psychiatrie und

Psychotherapie

Psychiatrische Universitätsklinik

Zürich

Lenggstrasse 31

CH-8032 Zürich

Tel. 0443842362

daniel.teichman@puk.zh.ch
Offensichtlich erachteten es die ersten jüdischen Surbtaler Studenten als wichtig, Studentenverbindungen beizutreten. Die Mitgliedschaft war ein deutliches Zeichen, Teil der damaligen Bürgergesellschaft zu werden und somit nicht nur formell, sondern auch kulturell zum «Studenten» zu werden. Tatsächlich gehörte die Mitgliedschaft in einer studentischen Verbindung neben der militärischen Laufbahn zu den wichtigsten Mitteln, um in der bürgerlichen Gesellschaft aufsteigen zu können.

Dabei fällt auf, dass jeder einen anderen Verein auswählte. Die Zofingia war 1819 als vaterländische Studentenverbindung in Zofingen (AG) gegründet worden. $1847 \mathrm{kam}$ es in Bern zum Bruch zwischen konservativen Führungspersönlichkeiten und fortschrittsorientierten Mitgliedern, worauf sich in mehreren Städten Sektionen eines Neuzofingervereins abspalteten. Es erstaunt nicht, dass sich der politisch liberal denkende Leman Bloch (*1827 Oberendingen, gest. 1902 Basel), der erste Schweizer Jude, der in Zürich Medizin studierte, 1851-1854 diesem zwischenzeitlich zu «Helvetia» umbenannten Verein anschloss.

In der Anfangszeit der Universität Zürich wurde das Duellieren noch geduldet, es wurde erst ab 1866 gesetzlich unter Strafe gestellt. Der Zofingerverein verbot daraufhin das Duell in seinen Reihen. Während Blochs Studienjahren war der Zofingerverein eine fakultativ schlagende Verbindung.

Der zehn Jahre später in Zürich immatrikulierte Raphael Bollag ( ${ }^{\star} 1839$ Endingen, gest. 1897 Lengnau) hingegen schloss sich der schlagenden Verbindung Tigurinia an, die 1850 gegründet

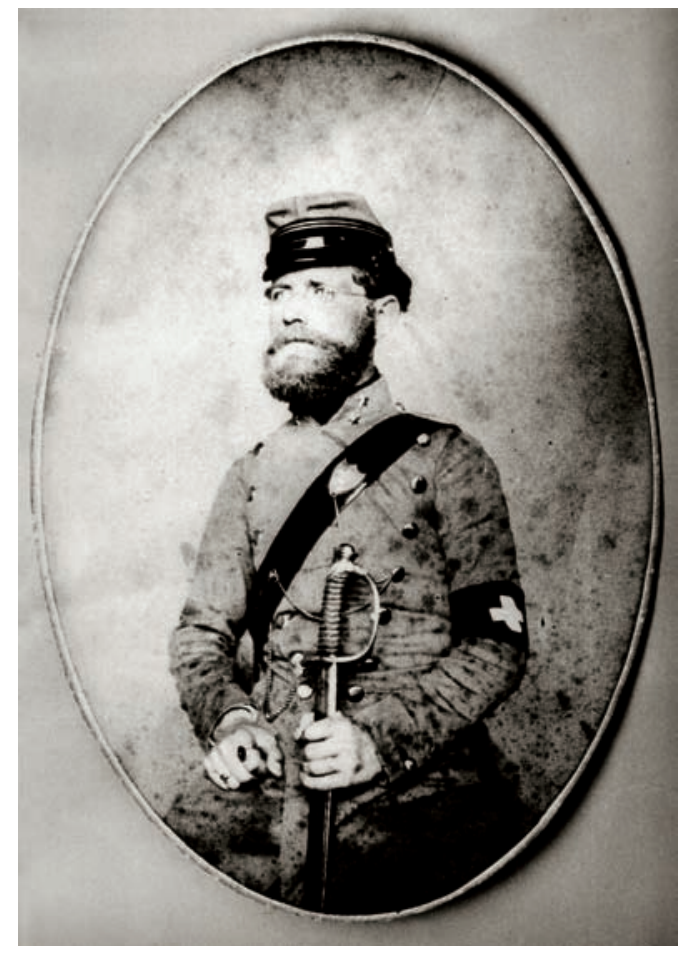

Leman Bloch als Hauptmann der Schweizer Armee (Privatbesitz Michael Bloch, Muttenz).

worden war und 1857 einen Mensurzwang eingeführt hatte. Raphael Bollag muss dieser Verbindung schon zu Beginn seines Studiums im Sommersemester 1861 beigetreten sein. Damals hatte die Verbindung acht Mitglieder, darunter war neben Bollag ein weiterer Jude, Paul Harrassowitz aus Berlin, der bei Bollags erstem Duell im Wald bei der Stefansburg als Sekundant fungierte. Gemäss Paukbuch, in dem sämtliche Fechtkämpfe festgehalten wurden, schlug Bollag ins- 


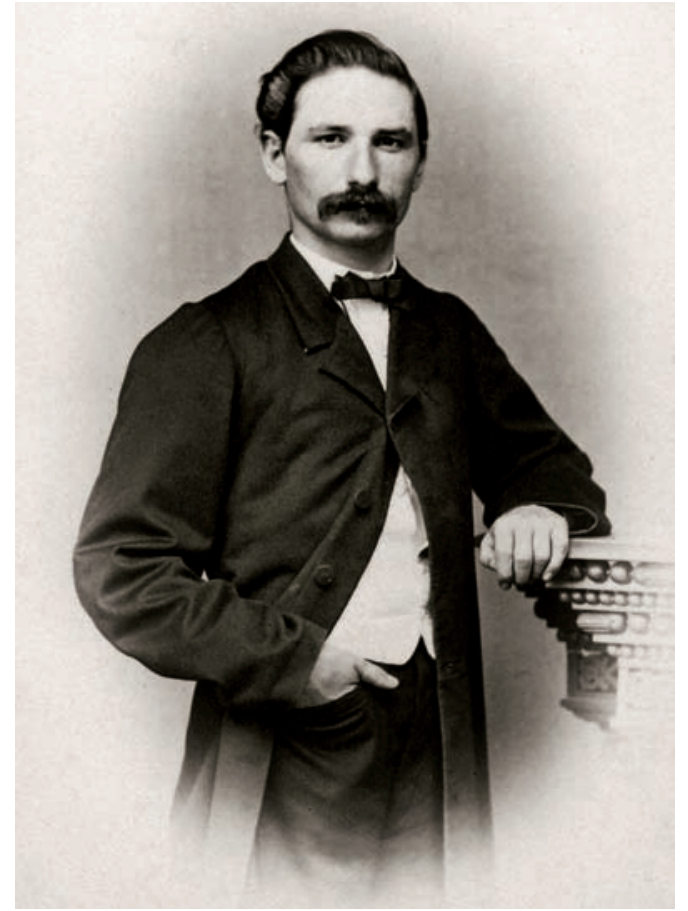

Raphael Bollag (undatierte Fotografie, Florence Guggenheim-Archiv, Aq 20a-c).

gesamt zehn Mensuren, die soweit eruierbar an Sonn- oder Wochentagen und damit nicht am Schabbat durchgeführt wurden. Ob er an den samstags abgehaltenen Pauktagen im Tiefenbrunnen teilnahm, ist nicht bekannt. Bereits nach dem zweiten Semester wurde Bollag «ohne Farben» aus der Tigurinia entlassen. Dies deutet darauf hin, dass er nicht in Eintracht mit der Verbindung ausschied.
Von den späteren jüdischen Medizinstudenten der Untersuchungsgruppe trat einzig noch Samuel Bollag (1859-1944, Stein-Säckingen im Fricktal) einem Verein bei, nämlich dem Universitätsturnverein. Die dort verbrachte Zeit muss für ihn eine wichtige Bedeutung gehabt haben, nahm er doch auch noch als alter Herr bis in seine letzten Lebensjahre regelmässig an den Veranstaltungen des später zur Verbindung «Utonia» gewordenen Vereins teil.

Dass die aus dem Surbtal stammenden Schweizer jüdischen Medizinstudenten in den Studentenverbindungen Zürichs nicht als Juden in Erscheinung treten wollten, sondern lieber als «normale» Schweizer Studenten, dafür sprechen Indizien aus der späteren Geschichte der jüdischen Studentenverbindungen. Ab 1910 wurden in Zürich nämlich, nicht zuletzt vor dem Hintergrund des zionistischen Gedankens, drei explizit jüdische Studentenverbindungen gegründet, die alle nur wenige Jahre Bestand hatten: Maccabea (1910-1912, fakultativ schlagend), Ivria (1914etwa 1919, schlagend) und Haschacher (1924etwa 1927, nichtschlagend). Unter ihren insgesamt 52 bekannten Mitgliedern befand sich lediglich ein Schweizer Bürger. Kein einziges Mitglied stammte aus dem Surbtal. Die Surbtaler jüdischen Studenten wollten sich offensichtlich nicht in «jüdischen» Verbindungen engagieren.

In der nächsten Folge: Visualisierung in der medizinischen Lehre am Beispiel der Dermatologie.
David Künzler,

Hausen am Albis

\section{Ungeheuer III}

(Ölplattform-Netz in der Nordsee)

Nur vom Flugzeug aus sozusagen mit dem Auge Gottes können wir das ungeheure Netz der ungeheuren Förderstelle sehen ungeheures Menschenwerk wir sehen ungeheuere Tentakel Polypenarme mit ungeheuren übergrossen Näpfen zum Saugen aus der Tiefe doch sieht die Phantasie auch ein Geflecht von grossen Nervenzellen verbunden mit Synapsen wo leider Öl statt Leben fliesst die Nervenzellen sind ohn' Geist und auch ohn' Seele ein Spiegel unsrer Zeit? wo Geist und Seele zu oft dem Geld geopfert werden in Öl zerfliessen ungeheure Ströme in Milliarden von Geld 